\title{
Three-particle correlation function in the electron-plasmon model
}

\author{
M.V.Vavrukh, S.B.Slobodyan, N.L.Tyshko \\ The Ivan Franko National University of Lviv, \\ Departament for Astrophysics, \\ 8 Kyrylo and Methodii Str., 79005 Lviv, Ukraine
}

Received August 3, 2005

\begin{abstract}
The method of calculating the $\boldsymbol{n}$-particle electron correlation functions for the electron-plasmon model is demonstrated. We have proposed this model earlier for the description of the strongly non-ideal electron liquid. The three-particle dynamic correlation function is calculated and presented in the elementary functions. The differences from the similar correlation function of the ordinary reference system approach in the electron liquid theory are investigated.
\end{abstract}

Key words: electron liquid, plasmon oscillation, $\boldsymbol{n}$-particle dynamic correlation functions, transition operator, collective variables

PACS: 05.30.Fk

\section{Introduction}

The practical calculations of the characteristics of degenerate electron systems are based on the perturbation theory over the power of the Coulomb potential which is connected with the local field conception. Since there is no rigorous microscopical theory of the local field correlation function, the development of alternative methods for description of the strongly non-ideal electrons systems remains one of the urgent tasks in statistical physics. It is well known that a very promissing direction in this field is a collective description of the interelectron interactions which presents the real situation, namely the existence of the collective motions. One of the earlier variants of such methods is described in the papers by Bohm and Pines [1-6]. This method uses a series of canonical transformations for the transition to the expanded space of the variables of electrons and plasmons. Consequently this approach bears an approximate character. Furthermore, at that time the problem of describing the strongly non-ideal systems was not considered to be urgent. Another variant of collective description was developed in the papers by Yukhnovskii et al. This is the method of displacements and collective variables (see [7-10]). In this variant of the collective description, as opposed to the approach by Bohm and Pines, the transition 
to the expanded space is made rigorously by means of a transition function. The absence of the divergent diagrams is characteristic of both approaches as opposed to the standard methods of perturbation theory.

The collective description has a deep physical basis as well as possesses some advantages over the other methods, especially in the strong non-ideality region. Based on the example of the electron liquid model in paper [11] a new variant of collective description is proposed, which differs from the variants by Bohm-Pines and from the method of the displacement and collective variables. We start with the secondary quantization representation. Transition to the expanded space is made using the transition operator which was introduced in paper [12]. These collective variables are an intermediate element in our approach. They serve for the introduction of the operators of the creation and destruction of plasmons. Partition function of the model in the electron and plasmon terms does not have any approximations. Perturbation theory relatively to the electron-plasmon interaction is built in terms of the $n$-particle dynamic correlation functions. Short-range interelectron interactions are taken into account in the local-field approximation [11].

\section{Correlation functions of the electron-plasmon model}

Due to the absence of the divergent diagrams the calculation of thermodynamic functions within the framework of the electron-plasmon model in the intermediate and strong non-ideality region is reduced to the calculation of only low order diagrams of the perturbation theory over the power of the operator of electron-plasmon interaction [11]. In these diagrams, the dynamic electron correlation functions, the so-called connected averages, are found, as in the following formulae:

$$
\eta_{n}\left(x_{1}, \ldots, x_{n}\right)=\left(\frac{\hbar^{2}}{2 m}\right)^{n} \varepsilon_{\mathrm{F}}^{-n} \beta^{-1}\left\langle T\left\{\hat{f}_{x_{1}} \hat{f}_{x_{2}} \cdots \hat{f}_{x_{n}}\right\}\right\rangle_{0}^{c}
$$

where $\varepsilon_{\mathrm{F}}=\hbar^{2} k_{\mathrm{F}}^{2} / 2 m$ is Fermi energy,

$$
\hat{f}_{x}=\sum_{\mathbf{k}, s} \sum_{\nu^{*}}(\mathbf{k q}) a_{\mathbf{k}+\mathbf{q}, s}^{+}\left(\nu^{*}+\nu\right) a_{\mathbf{k}, s}\left(\nu^{*}\right)
$$

$\nu_{n}^{*}=(2 n+1) \pi \beta^{-1}, \nu_{n}=2 \pi n \beta^{-1}$ are Matsubara frequencies, $\beta=\left(k_{\mathrm{B}} T\right)^{-1}, x \equiv$ $(\mathbf{q}, \nu), s$ is spin variable. Here, $a_{\mathbf{k}, s}\left(\nu^{*}\right)$ is superposition of the secondary quantization operators on the plane wave base in the interaction representation $[11,13]$

$$
a_{\mathbf{k}, s}\left(\nu^{*}\right)=\beta^{-\frac{1}{2}} \int_{0}^{\beta} a_{\mathbf{k}, s}\left(\beta^{\prime}\right) \exp \left(\mathrm{i} \nu^{*} \beta^{\prime}\right) \mathrm{d} \beta^{\prime} .
$$

Similar functions also appear in other approaches with renormalization of interactions (see [14]). The calculations of the functions of this type are not known in the literature. We shall show that functions $\eta_{n}\left(x_{1}, \ldots, x_{n}\right)$ can eventually lead to 
correlation functions which are constructed on the operator density of the particles $\hat{\rho}_{x}=\sum_{\mathbf{k}, s} \sum_{\nu^{*}} a_{\mathbf{k}+\mathbf{q}, s}^{+}\left(\nu^{*}+\nu\right) a_{\mathbf{k}, s}\left(\nu^{*}\right)$, namely

$$
\mu_{n}^{0}\left(x_{1}, \ldots, x_{n}\right)=\beta^{-1}\left\langle T\left\{\hat{\rho}_{x_{1}} \hat{\rho}_{x_{2}} \ldots \hat{\rho}_{x_{n}}\right\}\right\rangle_{0}^{c},
$$

which at $n \geqslant 3$ was originally calculated in papers $[14,15]$.

As it is shown in papers $[13,14]$

$$
-\left\langle T\left\{a_{\mathbf{k}_{1}, s_{1}}\left(\nu_{1}^{*}\right), a_{\mathbf{k}_{2}, s_{2}}^{+}\left(\nu_{2}^{*}\right)\right\}\right\rangle_{0}=G_{\mathbf{k}_{1}, s_{1}}^{e}\left(\nu_{1}^{*}\right) \delta_{\mathbf{k}_{1}, \mathbf{k}_{2}} \delta_{s_{1}, s_{2}} \delta_{\nu_{1}^{*}, \nu_{2}^{*}},
$$

where $G_{\mathbf{k}_{1}, s_{1}}^{e}\left(\nu_{1}^{*}\right)=\left\{\mathrm{i} \nu^{*}-\varepsilon_{\mathbf{k}}+\mu\right\}^{-1}$ is spectral representation of the one-particle Green's function of the reference system. Relationship (5) makes it possible to present functions $\eta_{n}\left(x_{1}, \ldots, x_{n}\right)$ in the form of such convolutions:

$$
\begin{aligned}
\eta_{2}\left(x_{1}, x_{2}\right)= & \beta^{-1} \delta_{x_{1}+x_{2}, 0}\left(\frac{\hbar^{2}}{2 m \varepsilon_{\mathrm{F}}}\right)^{2} \operatorname{Re} \sum_{\mathbf{k}, s} \sum_{\nu^{*}} G_{\mathbf{k}, s}^{e}\left(\nu^{*}\right) G_{\mathbf{k}+\mathbf{q}_{1}, s}^{e}\left(\nu^{*}+\nu_{1}\right) \\
& \times\left(\mathbf{k}, \mathbf{q}_{1}\right)\left(\mathbf{k}+\mathbf{q}_{1}, \mathbf{q}_{1}\right) ; \\
\eta_{3}\left(x_{1}, x_{2}, x_{3}\right)= & -2 \beta^{-1} \delta_{x_{1}+x_{2}+x_{3}, 0}\left(\frac{\hbar^{2}}{2 m \varepsilon_{\mathrm{F}}}\right)^{3} \operatorname{Re} \sum_{\mathbf{k}, s} \sum_{\nu^{*}} G_{\mathbf{k}, s}^{e}\left(\nu^{*}\right) \\
& \times G_{\mathbf{k}+\mathbf{q}_{1}, s}^{e}\left(\nu^{*}+\nu_{1}\right) G_{\mathbf{k}-\mathbf{q}_{2}, s}^{e}\left(\nu^{*}-\nu_{2}\right) \\
& \times\left(\mathbf{k}, \mathbf{q}_{1}\right)\left(\mathbf{k}+\mathbf{q}_{1}, \mathbf{q}_{1}+\mathbf{q}_{2}\right)\left(\mathbf{k}-\mathbf{q}_{2}, \mathbf{q}_{2}\right) ; \ldots,
\end{aligned}
$$

where symbol Re is applied to Bose-Matsubara frequencies $\left(\nu_{1}, \nu_{2}, \ldots\right)$. Functions $\eta_{n}\left(x_{1}, \ldots, x_{n}\right)$ are real functions of their arguments $\left(\mathbf{q}_{1}, \ldots, \mathbf{q}_{n} ; \nu_{1}, \ldots, \nu_{n}\right)$. Let us do the sums over the frequency $\nu^{*}$ using the rule [13]

$$
\beta^{-1} \sum_{\nu^{*}} G_{\mathbf{k}, s}^{e}\left(\nu^{*}\right)=n_{\mathbf{k}, s}=\left\{1+\exp \left[\beta\left(\varepsilon_{\mathbf{k}}-\mu^{*}\right)\right]\right\}^{-1}
$$

represented by $\eta_{n}\left(x_{1}, \ldots, x_{n}\right)$ in the form of the sum over the wave vector

$$
\begin{aligned}
\eta_{2}(x,-x)= & 2\left(\frac{\hbar^{2}}{2 m \varepsilon_{\mathrm{F}}}\right)^{2} \operatorname{Re} \sum_{\mathbf{k}, s} n_{\mathbf{k}, s} y(x)(\mathbf{k}, \mathbf{q})(\mathbf{k}+\mathbf{q}, \mathbf{q}) \\
\eta_{3}\left(x_{1}, x_{2}, x_{3}\right)= & -2 \delta_{x_{1}+x_{2}+x_{3}, 0}\left(\frac{\hbar^{2}}{2 m \varepsilon_{\mathrm{F}}}\right)^{3} \operatorname{Re} \sum_{\mathbf{k}, s} n_{\mathbf{k}, s} \\
& \times\left\{y\left(x_{1}\right) y\left(-x_{2}\right)\left(\mathbf{k}, \mathbf{q}_{1}\right)\left(\mathbf{k}-\mathbf{q}_{2}, \mathbf{q}_{2}\right)\left(\mathbf{k}+\mathbf{q}_{1}, \mathbf{q}_{1}+\mathbf{q}_{2}\right)\right. \\
& +y\left(x_{2}\right) y\left(-x_{3}\right)\left(\mathbf{k}, \mathbf{q}_{2}\right)\left(\mathbf{k}-\mathbf{q}_{3}, \mathbf{q}_{3}\right)\left(\mathbf{k}+\mathbf{q}_{2}, \mathbf{q}_{2}+\mathbf{q}_{3}\right) \\
& \left.+y\left(x_{3}\right) y\left(-x_{1}\right)\left(\mathbf{k}, \mathbf{q}_{3}\right)\left(\mathbf{k}-\mathbf{q}_{1}, \mathbf{q}_{1}\right)\left(\mathbf{k}+\mathbf{q}_{3}, \mathbf{q}_{3}+\mathbf{q}_{1}\right)\right\}
\end{aligned}
$$

where $y(x) \equiv\left\{\mathrm{i} \nu+\varepsilon_{\mathbf{k}}-\varepsilon_{\mathbf{k}+\mathbf{q}}\right\}^{-1}$. It should be noticed, that the expression in the brackets $(8)$ is symmetrical. The second and the third terms are obtained from the 
first one by means of cyclic transposition. Function $\eta_{2}(x,-x)$ is easily calculated. Transiting from the sum over the vector $\mathbf{k}$ to the integral and using spherical coordinate system (axis $0 \mathrm{z}$ is parallel to vector $\mathbf{q}$ ), at the absolute zero temperature we can obtain

$$
\begin{aligned}
\eta_{2}(x,-x) & =-\frac{N}{2 \varepsilon_{\mathrm{F}}} q^{2}+\left(u^{2}+\frac{1}{4} q^{2}\right) \mu_{2}^{0}(x,-x) \\
& =-\frac{N}{2 \varepsilon_{\mathrm{F}}} q^{2}\left\{1-3\left[u^{2}+\frac{1}{4} q^{2}\right] I_{2,0}(q, u)\right\},
\end{aligned}
$$

where $q \equiv|\mathbf{q}| k_{\mathrm{F}}^{-1} ; u \equiv \nu\left(2 \varepsilon_{\mathrm{F}} q\right)^{-1}, I_{2,0}(q, u)$ is the dimensionless function of these variables

$$
\begin{aligned}
I_{2,0}(q, u)= & \frac{1}{2}\left\{1+\frac{1}{2 q}\left(1+u^{2}-\frac{q^{2}}{4}\right) \sum_{\sigma= \pm 1} \sigma \ln \left[\left(1+\sigma \frac{q}{2}\right)^{2}+u^{2}\right]\right. \\
& \left.-u \sum_{\sigma= \pm 1} \arctan \left[\frac{1}{u}\left(1+\sigma \frac{q}{2}\right)\right]\right\} .
\end{aligned}
$$

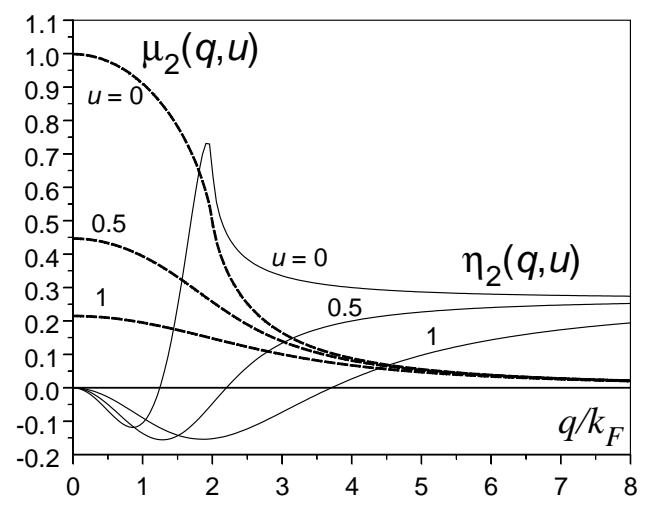

Figure 1. Two-particle correlation functions $\eta_{2}(q, u)=2 \varepsilon_{\mathrm{F}}(3 N)^{-1} \eta_{2}(x,-x)$ and $\mu_{2}(q, u)=2 \varepsilon_{\mathrm{F}}(3 N)^{-1} \mu_{2}^{0}(x,-x)$ at different values of the dimensionless frequency $(u=0 ; 0.5 ; 1)$.

Functions $\eta_{n}\left(x_{1}, \ldots, x_{n}\right)$ differ from $\mu_{n}^{0}\left(x_{1}, \ldots, x_{n}\right)$ due to the presence of the product of scalar factors $\left(\mathbf{k}, \mathbf{q}_{i}\right)$. But this "trifle" strongly complicates the calculation of the functions $\eta_{n}\left(x_{1}, \ldots, x_{n}\right)$ (at the $\left.n \geqslant 3\right)$ and forms the fundamental difference in the dependence of the function (1) and (4) from wave vectors $\mathbf{q}_{1}, \ldots, \mathbf{q}_{n}$, namely their asymptotes

$$
\begin{aligned}
\left|\mu_{n}^{0}\left(x_{1}, \ldots, x_{n}\right)\right| & \rightarrow \begin{cases}N \varepsilon_{\mathrm{F}}\left(\varepsilon_{\mathbf{q}_{1}} \ldots \varepsilon_{\mathbf{q}_{n}}\right)^{-1} & \text { at } q_{i} \gg k_{\mathrm{F}} ; \\
N \varepsilon_{\mathrm{F}}^{1-n} & \text { at } q_{i} \ll k_{\mathrm{F}} ; \nu_{i}=0 ;\end{cases} \\
\left|\eta_{n}\left(x_{1}, \ldots, x_{n}\right)\right| & \rightarrow \begin{cases}N \varepsilon_{\mathrm{F}}^{1-n} & \text { at } q_{i} \gg k_{\mathrm{F}} ; \\
N \varepsilon_{\mathrm{F}}^{1-n} q_{1} q_{2} \ldots q_{n} k_{\mathrm{F}}^{-n} & \text { at } q_{i} \ll k_{\mathrm{F}} ; \nu_{i}=0 .\end{cases}
\end{aligned}
$$

This asymptote is confirmed in figure 1 where dimensionless factors of the functions $\eta_{2}(x,-x)$ and $\mu_{2}(x,-x)$ as functions of wave vector $\mathbf{q}$ at the given frequencies are 
shown. Function $\eta_{2}(x,-x)$ is also an oscillating function in the region of the low and medium vectors, as opposed to $\mu_{2}^{0}(x,-x)$.

The calculation method of the correlation functions at the $n \geqslant 3$ is illustrated based on the example of three-particle function. At first, in each of the three terms of the formula (8) transformation is made to decrease the number of the scalar products by applying the identity type

$$
\begin{aligned}
\frac{\hbar^{2}}{2 m}\left(\mathbf{k}, \mathbf{q}_{1}\right) & =-\frac{1}{2}\left\{\varepsilon_{\mathbf{k}}+\varepsilon_{\mathbf{q}_{1}}-\varepsilon_{\mathbf{k}+\mathbf{q}_{1}}\right\}, \\
\frac{\hbar^{2}}{2 m}\left(\mathbf{k}-\mathbf{q}_{2}, \mathbf{q}_{2}\right) & =-\frac{1}{2}\left\{\varepsilon_{\mathbf{k}-\mathbf{q}_{2}}-\varepsilon_{\mathbf{k}}+\varepsilon_{\mathbf{q}_{2}}\right\} .
\end{aligned}
$$

Due to the transformations from each of the terms in formula (8) there arise components without energy denominators and components with one energy denominator type:

$$
\begin{aligned}
\mu_{2}(x) & =-2 \sum_{\mathbf{k}, s} n_{\mathbf{k}, s}\left[\mathrm{i} \nu+\varepsilon_{\mathbf{k}}-\varepsilon_{\mathbf{k}+\mathbf{q}}\right]^{-1} \\
\zeta_{2}\left(x_{1} \mid \mathbf{q}_{2}\right) & =-4 \sum_{\mathbf{k}, s} n_{\mathbf{k}, s} \frac{\hbar^{2}}{2 m}\left(\mathbf{k}, \mathbf{q}_{2}\right)\left[\mathrm{i} \nu_{1}+\varepsilon_{\mathbf{k}}-\varepsilon_{\mathbf{k}+\mathbf{q}_{1}}\right]^{-1}
\end{aligned}
$$

and components with two energy denominators but without the scalar products in the numerator of the fraction type

$$
\Gamma_{3}\left(x_{1} ;-x_{2}\right)=\sum_{\mathbf{k}, s} n_{\mathbf{k}, s}\left[\mathrm{i} \nu_{1}+\varepsilon_{\mathbf{k}}-\varepsilon_{\mathbf{k}+\mathbf{q}_{1}}\right]^{-1}\left[-\mathrm{i} \nu_{2}+\varepsilon_{\mathbf{k}}-\varepsilon_{\mathbf{k}-\mathbf{q}_{2}}\right]^{-1} .
$$

Calculation of the components of the type (13) is easily done by integrating over the vector $\mathbf{k}$ in the spherical coordinate system. In the dimensionless variables

$$
\begin{aligned}
\mu_{2}(x) & =\frac{3 N}{2 \varepsilon_{\mathrm{F}}} R_{2,0}(q, u), \\
\zeta_{2}\left(x \mid \mathbf{q}_{1}\right) & =\frac{3 N}{q^{2}}\left(\mathbf{q}, \mathbf{q}_{1}\right) C(q, u),
\end{aligned}
$$

where dimensionless complex functions $R_{2,0}(q, u)$ and $C(q, u)$ are shown in the Appendix. Introducing the notation $\varepsilon(q, u)=q^{2}+2 i u q$ we present $\eta_{3}\left(x_{1}, x_{2}, x_{3}\right)$ in the dimensionless variables as follows:

$$
\begin{aligned}
\eta_{3}\left(x_{1}, x_{2}, x_{3}\right)=\frac{3 N}{\left(2 \varepsilon_{\mathrm{F}}\right)^{2}} \delta_{\mathbf{q}_{1}+\mathbf{q}_{2}+\mathbf{q}_{3}, 0} \delta_{\nu_{1}+\nu_{2}+\nu_{3}, 0}\left\{-\frac{1}{3}\left(q_{1}^{2}+q_{2}^{2}+q_{3}^{2}\right)\right. \\
+\frac{1}{2} I_{2,0}\left(q_{1}, u_{1}\right) \operatorname{Re}\left[\varepsilon^{*}\left(q_{1}, u_{1}\right)\left(\varepsilon^{*}\left(q_{2}, u_{2}\right)+\varepsilon^{*}\left(q_{3}, u_{3}\right)\right)\right] \\
+\frac{1}{2} I_{2,0}\left(q_{2}, u_{2}\right) \operatorname{Re}\left[\varepsilon^{*}\left(q_{2}, u_{2}\right)\left(\varepsilon^{*}\left(q_{3}, u_{3}\right)+\varepsilon^{*}\left(q_{1}, u_{1}\right)\right)\right] \\
+\frac{1}{2} I_{2,0}\left(q_{3}, u_{3}\right) \operatorname{Re}\left[\varepsilon^{*}\left(q_{3}, u_{3}\right)\left(\varepsilon^{*}\left(q_{1}, u_{1}\right)+\varepsilon^{*}\left(q_{2}, u_{2}\right)\right)\right]
\end{aligned}
$$




$$
\begin{aligned}
& -\frac{\operatorname{Re}}{4}\left[R_{2,0}\left(q_{1}, u_{1}\right)\left(\varepsilon\left(q_{1}, u_{1}\right) \varepsilon\left(q_{3}, u_{3}\right)+\varepsilon^{*}\left(q_{1}, u_{1}\right) \varepsilon\left(q_{2}, u_{2}\right)\right)\right] \\
& -\frac{\operatorname{Re}}{4}\left[R_{2,0}\left(q_{2}, u_{2}\right)\left(\varepsilon\left(q_{2}, u_{2}\right) \varepsilon\left(q_{1}, u_{1}\right)+\varepsilon^{*}\left(q_{2}, u_{2}\right) \varepsilon\left(q_{3}, u_{3}\right)\right)\right] \\
& -\frac{\operatorname{Re}}{4}\left[R_{2,0}\left(q_{3}, u_{3}\right)\left(\varepsilon\left(q_{3}, u_{3}\right) \varepsilon\left(q_{2}, u_{2}\right)+\varepsilon^{*}\left(q_{3}, u_{3}\right) \varepsilon\left(q_{1}, u_{1}\right)\right)\right] \\
& -\frac{\left(\mathbf{q}_{1}, \mathbf{q}_{2}\right)}{2} \operatorname{Re}\left[\varepsilon^{*}\left(q_{1}, u_{1}\right) C\left(q_{1}, u_{1}\right) q_{1}^{-2}+\varepsilon^{*}\left(q_{2}, u_{2}\right) C^{*}\left(q_{2}, u_{2}\right) q_{2}^{-2}\right] \\
& -\frac{\left(\mathbf{q}_{2}, \mathbf{q}_{3}\right)}{2} \operatorname{Re}\left[\varepsilon^{*}\left(q_{2}, u_{2}\right) c\left(q_{2}, u_{2}\right) q_{2}^{-2}+\varepsilon^{*}\left(q_{3}, u_{3}\right) c^{*}\left(q_{3}, u_{3}\right) q_{3}^{-2}\right] \\
& \left.-\frac{\left(\mathbf{q}_{1}, \mathbf{q}_{3}\right)}{2} \operatorname{Re}\left[\varepsilon^{*}\left(q_{3}, u_{3}\right) C\left(q_{3}, u_{3}\right) q_{3}^{-2}+\varepsilon^{*}\left(q_{1}, u_{1}\right) C^{*}\left(q_{1}, u_{1}\right) q_{1}^{-2}\right]\right\} \\
& -\frac{1}{4} \delta_{\mathbf{q}_{1}+\mathbf{q}_{2}+\mathbf{q}_{3}, 0} \delta_{\nu_{1}+\nu_{2}+\nu_{3}, 0} \operatorname{Re}\left\{\varepsilon^{*}\left(q_{1}, u_{1}\right) \varepsilon^{*}\left(q_{2}, u_{2}\right) \varepsilon^{*}\left(q_{3}, u_{3}\right)\right. \\
& \left.\times\left[\Gamma_{3}\left(x_{1} ;-x_{2}\right)+\Gamma_{3}\left(x_{2} ;-x_{3}\right)+\Gamma_{3}\left(x_{3} ;-x_{1}\right)\right]\right\} .
\end{aligned}
$$

The last term in formula (16) is connected with the function $\mu_{3}^{0}\left(x_{1}, x_{2}, x_{3}\right)$, since

$$
\begin{aligned}
\mu_{3}^{0}\left(x_{1}, x_{2}, x_{3}\right)= & -2 \delta_{\mathbf{q}_{1}+\mathbf{q}_{2}+\mathbf{q}_{3}, 0} \delta_{\nu_{1}+\nu_{2}+\nu_{3}, 0} \operatorname{Re}\left\{\Gamma_{3}\left(x_{1} ;-x_{2}\right)\right. \\
& \left.+\Gamma_{3}\left(x_{2} ;-x_{3}\right)+\Gamma_{3}\left(x_{3} ;-x_{1}\right)\right\} .
\end{aligned}
$$

The difficulty in calculating $\Gamma_{3}\left(x_{i} ;-x_{j}\right)$ as well as the functions of higher order (together with a greater number of energy denominators) is caused by the necessity to integrate over the vector $\mathbf{k}$ at the given configuration of the vectors $\mathbf{q}_{1}, \ldots, \mathbf{q}_{n}$. However, the integration over the angled variables of the vector $\mathbf{k}$ is easily done if one uses Feynman identity [16],

$$
\prod_{j=1}^{n} A_{j}^{-1}=(n-1) ! \int_{0}^{1} \ldots \int_{0}^{1} \mathrm{~d} \alpha_{1} \ldots \mathrm{d} \alpha_{n}\left\{\sum_{j=1}^{n} \alpha_{j} A_{j}\right\}^{-n} \delta\left(\sum_{j=1}^{n} \alpha_{j}-1\right) .
$$

Let us consider the calculation function $\Gamma_{3}\left(x_{1} ; x_{2}\right)$ for the case of frequencies $\nu_{1}$ and $\nu_{2}$ of the same sign, since real part $\sum_{j} \alpha_{j} A_{j}$ can be equal to zero at some values $\alpha$. Then, there appears a condition of positive distinctness of the imaginary part $\sum_{j} \alpha_{j} A_{j}$. The formulae which permit to obtain the function $\Gamma_{3}\left(x_{1} ; x_{2}\right)$ for frequencies of different sign are given in the Appendix. In formula (14) we transit to dimensionless variables $q_{i}=\left|\mathbf{q}_{i}\right| k_{\mathrm{F}}^{-1}, u_{i}=\nu_{i}\left(2 \varepsilon_{\mathrm{F}} q_{i}\right)^{-1}$ and use the identity (18) at $n=2$. Thus, we obtain the following representation

$$
\begin{aligned}
\Gamma_{3}\left(x_{1} ; x_{2}\right) & =\frac{3 N}{4 \pi q_{1} q_{2}\left(2 \varepsilon_{\mathrm{F}}\right)^{2}} \int \mathrm{d} \mathbf{k} n_{\mathbf{k}, s} \int_{0}^{1} \mathrm{~d} \alpha F_{\alpha}^{-2} \\
F_{\alpha} & =\alpha\left[\left(\mathbf{k}, \mathbf{e}_{1}\right)+\xi_{1}\right]+(1-\alpha)\left[\left(\mathbf{k}, \mathbf{e}_{2}\right)+\xi_{2}\right]
\end{aligned}
$$


where $\mathbf{e}_{i}=\mathbf{q}_{i}\left|\mathbf{q}_{i}\right|^{-1}$, vector $\mathbf{k}$ measured pin the units of $k_{\mathrm{F}}, \xi_{j}=\frac{1}{2} q_{j}-\mathrm{i} u_{j} ; j=1,2$. Let us introduce vector

$$
\boldsymbol{\rho}_{\alpha}=\alpha \mathbf{e}_{1}+(1-\alpha) \mathbf{e}_{2}
$$

and mark

$$
\begin{aligned}
& \Omega_{\alpha}=\alpha\left(\xi_{2}-\xi_{1}\right)-\xi_{2}=\Omega_{\alpha}^{c}-\mathrm{i} \Omega_{\alpha}^{s} ; \\
& \Omega_{\alpha}^{c}=\frac{1}{2}\left[\alpha\left(q_{1}-q_{2}\right)+q_{2}\right], \quad \Omega_{\alpha}^{s}=\alpha\left(u_{1}-u_{2}\right)+u_{2},
\end{aligned}
$$

so that $\Omega_{\alpha}^{c}, \Omega_{\alpha}^{s} \geqslant 0$. In these notations

$$
F_{\alpha}=\left(\mathbf{k}, \boldsymbol{\rho}_{\alpha}\right)+\Omega_{\alpha},
$$

therefore integration over the angled variables of the vector $\mathbf{k}$ is done in the spherical coordinate system (axis $0 z$ parallel to vector $\boldsymbol{\rho}_{\alpha}$ ). After integrating over the module of the vector $\mathbf{k}$ we obtain $\Gamma_{3}\left(x_{1} ; x_{2}\right)$ in the form of single integral over the parameter $\alpha$

$$
\begin{aligned}
\Gamma_{3}\left(x_{1} ; x_{2}\right) & =-\frac{3 N}{q_{1} q_{2}\left(2 \varepsilon_{\mathrm{F}}\right)^{2}} \int_{0}^{1} \frac{\mathrm{d} \alpha}{\rho_{\alpha}^{2}}\left\{1-\frac{\Omega_{\alpha}}{2 \rho_{\alpha}} \ln \left|\frac{\rho_{\alpha}+\Omega_{\alpha}}{\rho_{\alpha}-\Omega_{\alpha}}\right|\right\}, \\
\rho_{\alpha} & \equiv\left|\boldsymbol{\rho}_{\alpha}\right|=\left\{1-2 \alpha(1-t)+2 \alpha^{2}(1-t)\right\}^{\frac{1}{2}}
\end{aligned}
$$

$t \equiv t_{12} \equiv\left(\mathbf{e}_{1}, \mathbf{e}_{2}\right)$ is cosine of the angle between vectors $\mathbf{q}_{1}$ and $\mathbf{q}_{2}$.

The integral over the variable $\alpha$ is divided into two ones. The integral in which we have logarithm is integrated by parts. Then we unite it with the integral in which the subintegral function is equal to $\rho_{\alpha}^{-2}$. Thus, the integral (23) is considerably simplified

$$
\begin{aligned}
\Gamma_{3}\left(x_{1} ; x_{2}\right)= & \frac{3 N}{\left(2 \varepsilon_{\mathrm{F}}\right)^{2}}\left[2 q_{1} q_{2}\left(1-t^{2}\right)\right]^{-1} \\
& \times\left\{\left[\xi_{2}-t \xi_{1}\right] \ln \left[\frac{1-\xi_{1}}{1+\xi_{1}}\right]+\left[\xi_{1}-t \xi_{2}\right] \ln \left[\frac{1-\xi_{2}}{1+\xi_{2}}\right]\right\} \\
& -\frac{3 N}{\left(2 \varepsilon_{\mathrm{F}}\right)^{2}}\left[2 q_{1} q_{2}\left(1-t^{2}\right)\right]^{-1} \delta(\xi, t) \int_{0}^{1} \frac{\mathrm{d} \alpha}{\rho_{\alpha}^{2}-\Omega_{\alpha}^{2}}
\end{aligned}
$$

where

$$
\begin{aligned}
\delta(\xi, t) & =1-t^{2}-\xi_{1}^{2}-\xi_{2}^{2}+2 t \xi_{1} \xi_{2} \\
\rho_{\alpha}^{2}-\Omega_{\alpha}^{2} & =\alpha^{2}\left\{2(1-t)-\left(\xi_{1}-\xi_{2}\right)^{2}\right\}+2 \alpha\left\{\xi_{2}\left(\xi_{2}-\xi_{1}\right)-(1-t)\right\}+1-\xi_{2}^{2} .
\end{aligned}
$$

Let $\alpha_{1}=\alpha_{1}^{c}+\mathrm{i} \alpha_{1}^{s}, \alpha_{2}=\alpha_{2}^{c}+\mathrm{i} \alpha_{2}^{s}$ are roots of the equation $\rho_{\alpha}^{2}-\Omega_{\alpha}^{2}=0$ :

$$
\begin{aligned}
& \alpha_{1}^{c}=\left\{p_{c}^{2}+p_{s}^{2}\right\}^{-1}\left\{p_{c}\left(b_{c}-\eta\right)+p_{s}\left(b_{s}+\zeta\right)\right\} ; \\
& \alpha_{1}^{s}=\left\{p_{c}^{2}+p_{s}^{2}\right\}^{-1}\left\{p_{c}\left(b_{s}+\zeta\right)-p_{s}\left(b_{c}-\eta\right)\right\} ; \\
& \alpha_{2}^{c}=\left\{p_{c}^{2}+p_{s}^{2}\right\}^{-1}\left\{p_{c}\left(b_{c}+\eta\right)+p_{s}\left(b_{s}-\zeta\right)\right\} ; \\
& \alpha_{2}^{s}=\left\{p_{c}^{2}+p_{s}^{2}\right\}^{-1}\left\{p_{c}\left(b_{s}-\zeta\right)-p_{s}\left(b_{c}+\eta\right)\right\} .
\end{aligned}
$$


Here the following notations are used

$$
\begin{aligned}
p_{c} & =2(1-t)-\frac{1}{4}\left(q_{1}-q_{2}\right)^{2}+\left(u_{1}-u_{2}\right)^{2} ; \\
p_{s} & =\left(q_{1}-q_{2}\right)\left(u_{1}-u_{2}\right) ; \\
b_{c} & =1-t-u_{2}\left(u_{1}-u_{2}\right)+\frac{q_{2}}{4}\left(q_{1}-q_{2}\right) ; \\
b_{s} & =\frac{u_{2}}{2}\left(q_{2}-q_{1}\right)-\frac{q_{2}}{2}\left(u_{1}-u_{2}\right) ; \quad[\delta(\xi, t)]^{\frac{1}{2}}=\zeta+\mathrm{i} \eta ; \\
\zeta & =\frac{1}{\sqrt{2}}\left\{\delta_{c}+\left[\delta_{c}^{2}+\delta_{s}^{2}\right]^{\frac{1}{2}}\right\}^{\frac{1}{2}} ; \quad \eta=\frac{\delta_{s}}{\sqrt{2}}\left\{\delta_{c}+\left[\delta_{c}^{2}+\delta_{s}^{2}\right]^{\frac{1}{2}}\right\}^{-\frac{1}{2}} ; \\
\delta_{c} & =1-t^{2}-\frac{1}{4}\left(q_{1}^{2}+q_{2}^{2}-2 t q_{1} q_{2}\right)+\left(u_{1}^{2}+u_{2}^{2}-2 t u_{1} u_{2}\right) ; \\
\delta_{s} & =u_{1}\left(q_{1}-t q_{2}\right)+u_{2}\left(q_{2}-t q_{1}\right) .
\end{aligned}
$$

Let us divide the subintegral function into simple factors and present the integral over the variable $\alpha$ in the following form:

$$
\begin{aligned}
& \delta(\xi, t) \int_{0}^{1} \frac{\mathrm{d} \alpha}{\rho_{\alpha}^{2}-\Omega_{\alpha}^{2}}=\gamma_{3}^{c}+\mathrm{i} \gamma_{3}^{s}, \\
& \gamma_{3}^{c}=\frac{\zeta}{2}\left(\tilde{A}_{1}-\tilde{A}_{2}\right)+\frac{\eta}{4}\left(\tilde{L}_{1}-\tilde{L}_{2}\right) ; \quad \gamma_{3}^{s}=\frac{\eta}{2}\left(\tilde{A}_{1}-\tilde{A}_{2}\right)-\frac{\zeta}{4}\left(\tilde{L}_{1}-\tilde{L}_{2}\right) ; \\
& \tilde{A}_{i}=\arctan \frac{1-\alpha_{i}^{c}}{\alpha_{i}^{s}}+\arctan \frac{\alpha_{i}^{c}}{\alpha_{i}^{s}} ; \quad \tilde{L}_{i} \equiv \ln \frac{\left(1-\alpha_{i}^{c}\right)^{2}+\left(\alpha_{i}^{s}\right)^{2}}{\left(\alpha_{i}^{c}\right)^{2}+\left(\alpha_{i}^{s}\right)^{2}} ; \quad(i=1,2) .
\end{aligned}
$$

Thus, the real and the imaginary components of the function $\Gamma_{3}\left(x_{1} ; x_{2}\right)$ are determined in the following expressions:

$$
\begin{aligned}
\Gamma_{3}^{c}\left(x_{1} ; x_{2}\right)= & \frac{3 N}{\left(2 \varepsilon_{\mathrm{F}}\right)^{2}}\left[2 q_{1} q_{2}\left(1-t^{2}\right)\right]^{-1}\left\{-\frac{1}{4}\left(q_{2}-t q_{1}\right) L\left(q_{1}, u_{1}\right)-\left(u_{2}-t u_{1}\right)\right. \\
& \left.\times A\left(q_{1}, u_{1}\right)-\frac{1}{4}\left(q_{1}-t q_{2}\right) L\left(q_{2}, u_{2}\right)-\left(u_{1}-t u_{2}\right) A\left(q_{2}, u_{2}\right)-\gamma_{3}^{c}\right\} ; \\
\Gamma_{3}^{s}\left(x_{1} ; x_{2}\right)= & -\frac{3 N}{\left(2 \varepsilon_{\mathrm{F}}\right)^{2}}\left[2 q_{1} q_{2}\left(1-t^{2}\right)\right]^{-1} \frac{1}{2}\left\{\left(q_{2}-t q_{1}\right) A\left(q_{1}, u_{1}\right)-\left(u_{2}-t u_{1}\right)\right. \\
& \left.\times L\left(q_{1}, u_{1}\right)+\left(q_{1}-t q_{2}\right) A\left(q_{2}, u_{2}\right)-\left(u_{1}-t u_{2}\right) L\left(q_{2}, u_{2}\right)+\gamma_{3}^{s}\right\}
\end{aligned}
$$

where functions $A(q, u)$ and $L(q, u)$ are shown in the Appendix.

Expressions (15), (16), (26)-(29) together with the formulae from the Appendix determine the function $\eta_{3}\left(x_{1}, x_{2}, x_{3}\right)$.

\section{Conclusions}

The proposed method for calculating the three-particle dynamic correlation function permits to calculate the degenerate function of the fourth order $\eta_{4}\left(x_{1},-x_{1}, x_{2},-x_{2}\right)$ 
in the analytical form. Function $\eta_{4}\left(x_{1}, x_{2}, x_{3},-x_{1}-x_{2}-x_{3}\right)$ and function $\eta_{5}\left(x,-x, x_{1}, x_{2},-x_{1}-x_{2}\right)$ can be presented in the form of single integral over the parameter $\alpha$ from elementary functions. Due to a good fit of the series of the perturbation theory there is no need in calculating the functions $\eta_{n}\left(x_{1}, \ldots, x_{n}\right)$ of the higher order.

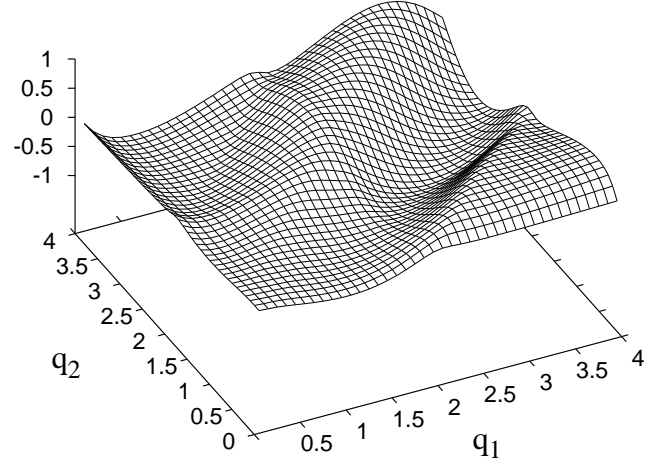

(a)

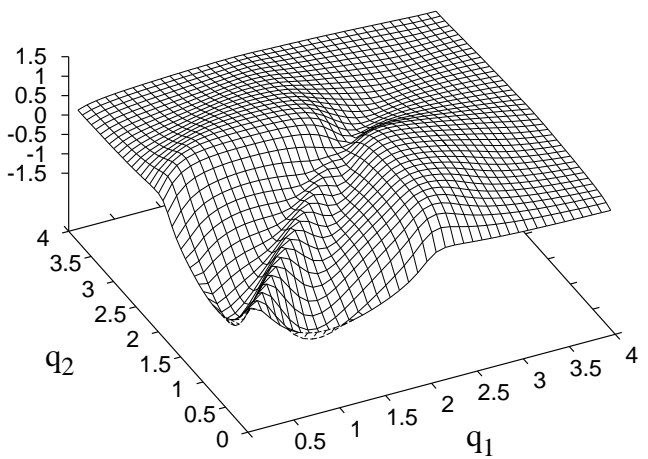

(b)

Figure 2. Functions $\eta_{3}\left(x_{1}, x_{2}, x_{3}\right)$ (figure 2a) and $\mu_{3}^{0}\left(x_{1}, x_{2}, x_{3}\right)$ (figure $2 \mathrm{~b}$ ) at the $\overline{\nu_{1}}=\overline{\nu_{2}}=0,1 ; t_{12}=-1\left(\bar{\nu}=\nu\left(2 \varepsilon_{\mathrm{F}}\right)^{-1}\right)$.

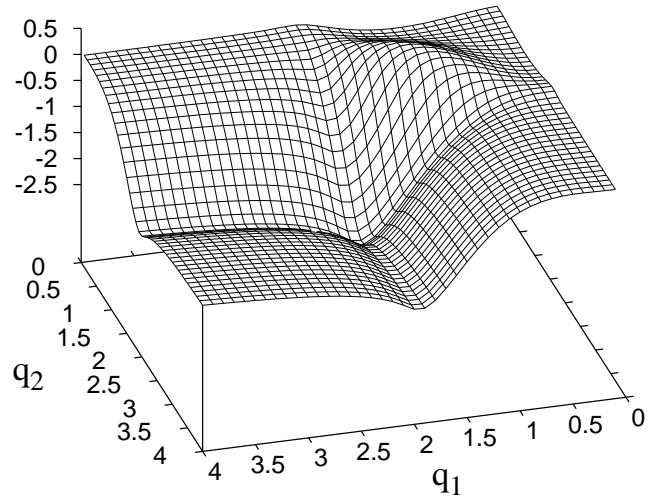

(a)

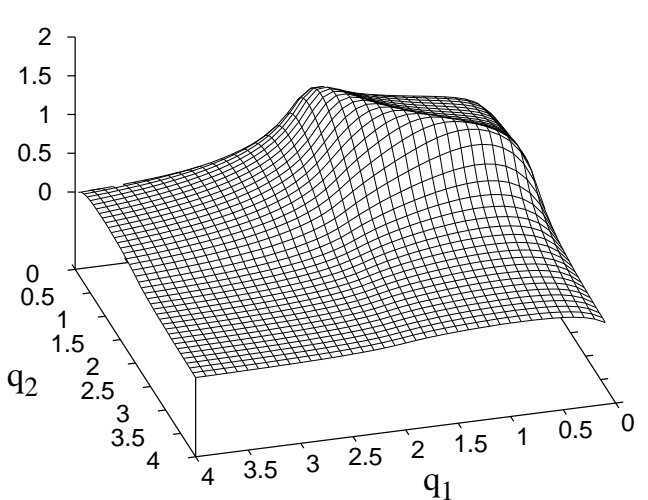

(b)

Figure 3. Functions $\eta_{3}\left(x_{1}, x_{2}, x_{3}\right)$ (figure $3 \mathrm{a}$ ) and $\mu_{3}^{0}\left(x_{1}, x_{2}, x_{3}\right)$ (figure $3 \mathrm{~b}$ ) at the $\overline{\nu_{1}}=\overline{\nu_{2}}=0,1 ; t_{12}=0$.

Figure 1 and figures 2-6 clearly show that the function $\eta_{3}\left(x_{1}, x_{2}, x_{3}\right)$ differs from the correlation function of the ordinary reference system approach $\mu_{3}^{0}\left(x_{1}, x_{2}, x_{3}\right)$. In the region of the small wave numbers the $\eta_{3}\left(x_{1}, x_{2}, x_{3}\right)$ have much smaller values (in absolute value) than $\mu_{3}^{0}\left(x_{1}, x_{2}, x_{3}\right)$. As a rule, $\eta_{3}\left(x_{1}, x_{2}, x_{3}\right)$ is the oscillating function (even in those cases when $\mu_{3}^{0}\left(x_{1}, x_{2}, x_{3}\right)$ have constant signs (see figures $3-$ $5)$ ). Similarly to $\mu_{3}^{0}\left(x_{1}, x_{2}, x_{3}\right)$, the functions $\eta_{3}\left(x_{1}, x_{2}, x_{3}\right)$ have a strong frequency dependence. At the frequencies $\nu_{1}=\nu_{2}$, the functions $\mu_{3}^{0}\left(x_{1}, x_{2}, x_{3}\right)$ are the surfaces that are symmetrical relatively to the trussed $q_{1}=q_{2}$ at any values of $\cos \left(\mathbf{q}_{1}, \mathbf{q}_{2}\right)$ (figures $2 \mathrm{~b}, 3 \mathrm{~b}, 4 \mathrm{~b})$. At $\nu_{1} \neq \nu_{2}$ the functions $\mu_{3}^{0}\left(x_{1}, x_{2}, x_{3}\right)$ are asymmetrical. 


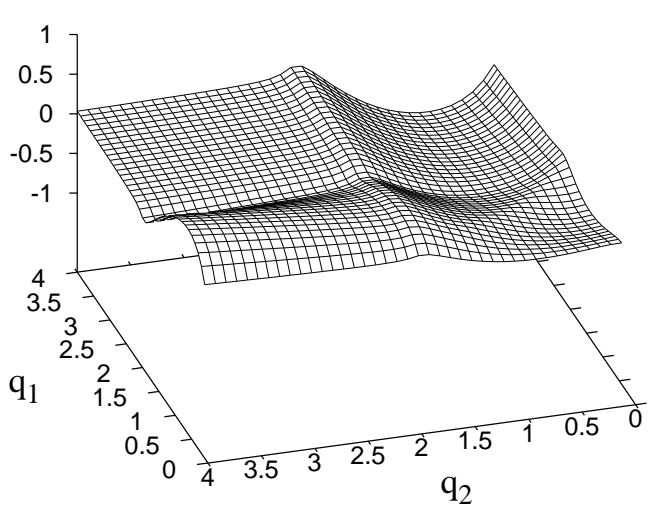

(a)

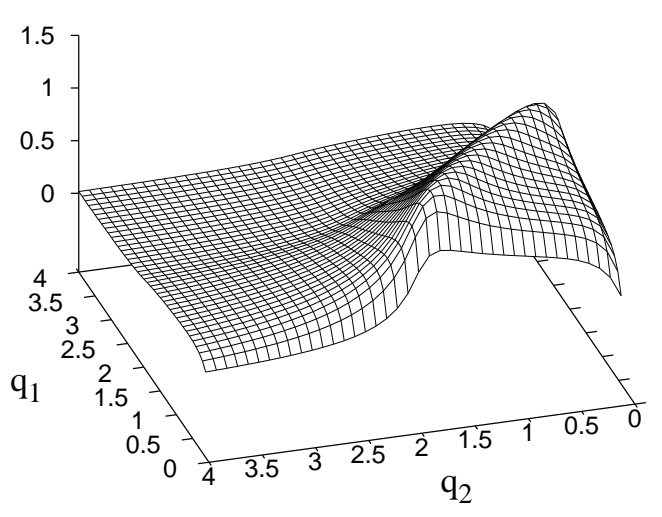

(b)

Figure 4. Functions $\eta_{3}\left(x_{1}, x_{2}, x_{3}\right)$ (figure $4 \mathrm{a}$ ) and $\mu_{3}^{0}\left(x_{1}, x_{2}, x_{3}\right)$ (figure $4 \mathrm{~b}$ ) at the $\overline{\nu_{1}}=\overline{\nu_{2}}=0,1 ; t_{12}=1$.

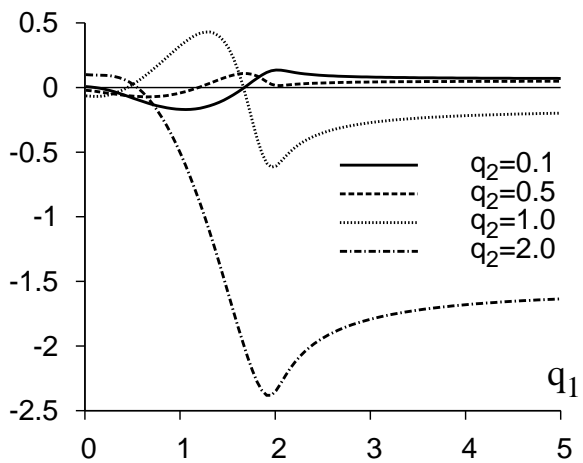

(a)

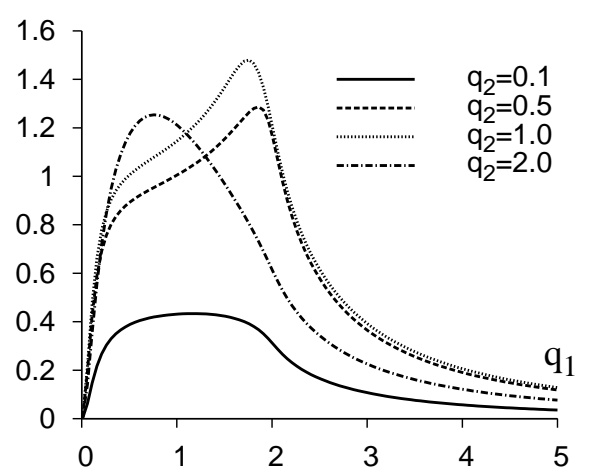

(b)

Figure 5. Functions $\eta_{3}\left(x_{1}, x_{2}, x_{3}\right)$ (figure 5a) and $\mu_{3}^{0}\left(x_{1}, x_{2}, x_{3}\right)$ (figure $5 \mathrm{~b}$ ) at the constant waves numbers $q_{2}$ for the case where $\overline{\nu_{1}}=\overline{\nu_{2}}=0,1 ; t_{12}=0\left(q_{2}=\right.$ $0,1 ; 0,5 ; 1 ; 2)$.

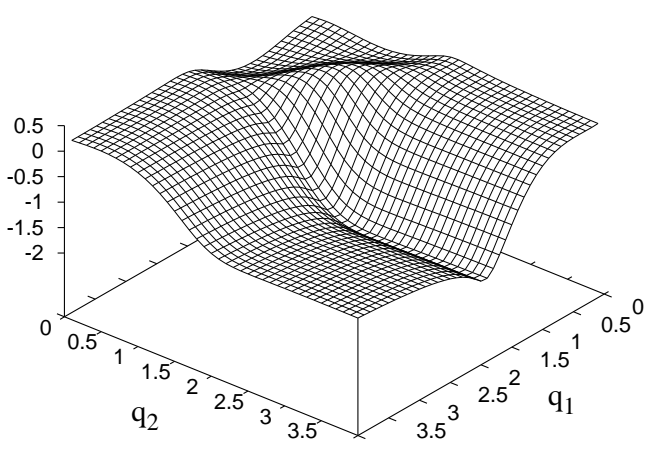

(a)

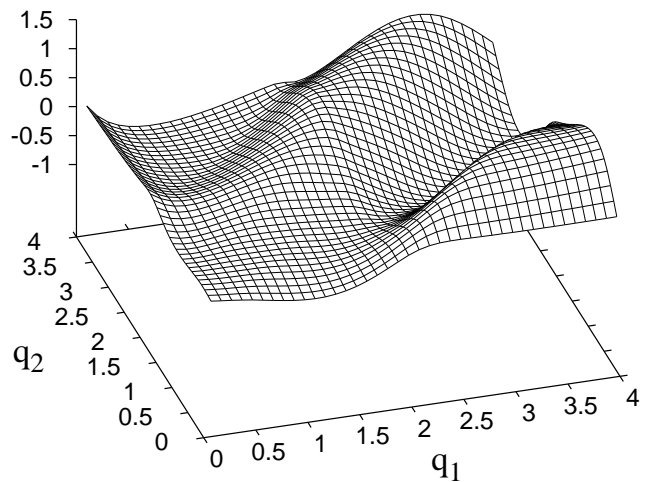

(b)

Figure 6. Function $\eta_{3}\left(x_{1}, x_{2}, x_{3}\right)$ for the case of unequal frequencies $\left(\overline{\nu_{1}}=\right.$ 0,$\left.1 ; \overline{\nu_{2}}=0,3\right)$ at the $t_{12}=0$. 
The functions $\eta_{3}\left(x_{1}, x_{2}, x_{3}\right)$ have a strong dependence on $\cos \left(\mathbf{q}_{1}, \mathbf{q}_{2}\right)$ and are asymmetrical even at the $\nu_{1}=\nu_{2}$. This is illustrated in figures $2 \mathrm{a}, 3 \mathrm{a}$, 4a. From figures $2-7$, it arises that the asymptotes of the functions $\mu_{3}^{0}\left(x_{1}, x_{2}, x_{3}\right)$ and $\eta_{3}\left(x_{1}, x_{2}, x_{3}\right)$ correspond to the formula (11).

\section{Appendix}

Here we introduce the explicit expressions for the functions (13) in the dimensionless variables $q=|\mathbf{q}| k_{\mathrm{F}}^{-1}, u=\nu\left(2 \varepsilon_{\mathrm{F}} q\right)^{-1}$ :

$$
\begin{aligned}
\mu_{2}(x) & =\frac{3 N}{2 \varepsilon_{\mathrm{F}}} R_{2,0}(q, u), \\
R_{2,0}(q, u) & =I_{2,0}(q, u)+\mathrm{i} J_{2,0}(q, u),
\end{aligned}
$$

where $I_{2,0}(q, u)$ is determined by formula (10),

$$
\begin{aligned}
J_{2,0}(q, u) & =-\frac{1}{2 q}\left\{u-\frac{1}{2}\left(1+u^{2}-\frac{q^{2}}{2}\right) A(q, u)\right\} ; \quad \zeta_{2}\left(x \mid \mathbf{q}_{1}\right)=\frac{3 N}{q^{2}}\left(\mathbf{q}, \mathbf{q}_{1}\right) C(q, u) \\
C(q, u) & =C^{c}(q, u)+\mathrm{i} C^{s}(q, u) \\
C^{c}(q, u) & =\frac{2}{3}+\left(u^{2}-\frac{q^{2}}{4}\right)-\frac{q}{8}\left(1+3 u^{2}-\frac{q^{2}}{4}\right) L(q, u)-\frac{u}{2}\left(1+u^{2}-\frac{3}{4} q^{2}\right) A(q, u) \\
C^{s}(q, u) & =q u+\frac{u}{4}\left(1+u^{2}-\frac{3}{4} q^{2}\right) L(q, u)-\frac{1}{4} q\left(1+3 u^{2}-\frac{q^{2}}{4}\right) A(q, u) .
\end{aligned}
$$

Above we use the following marks:

$$
L(q, u)=\ln \frac{u^{2}+\left(1+\frac{q}{2}\right)^{2}}{u^{2}+\left(1-\frac{q}{2}\right)^{2}} ; \quad A(q, u)=\arctan \frac{1+\frac{q}{2}}{u}+\arctan \frac{1-\frac{q}{2}}{u} .
$$

Proceeding from the definition (14) and the explicit expression $\Gamma_{3}\left(x_{1} ; x_{2}\right) \equiv$ $\Gamma_{3}\left(\mathbf{q}_{1}, \nu_{1} ; \mathbf{q}_{2}, \nu_{2}\right)$ at the $\nu_{1}, \nu_{2} \geqslant 0$ or $\nu_{1}, \nu_{2}<0$ by way of elementary transformations we can obtain such relationships which permit to write $\Gamma_{3}\left(\mathbf{q}_{1}, \nu_{1} ; \mathbf{q}_{2}, \nu_{2}\right)$ for positive and negative wave vectors and frequencies. Let $\Gamma_{3}\left(x_{1} ; x_{2}\right)$ for the case of the frequencies of the same sign $\nu_{1}, \nu_{2}$ be written in the dimensionless form:

$$
\Gamma_{3}\left(x_{1} ; x_{2}\right) \equiv \frac{3 N}{\left(2 \varepsilon_{\mathrm{F}}\right)^{2}} \gamma_{3}\left(q_{1}, q_{2}, t \mid \frac{\overline{\nu_{1}}}{q_{1}}, \frac{\overline{\nu_{2}}}{\overline{q_{2}}}\right)
$$

where $t \equiv t_{12}=\cos \left(\widehat{\mathbf{q}_{1}, \mathbf{q}_{2}}\right), \overline{\nu_{i}} \equiv \nu\left(2 \varepsilon_{\mathrm{F}}\right)^{-1}$.

Then

$$
\begin{aligned}
\Gamma_{3}\left(\mathbf{q}_{1}, \nu_{1} ;-\mathbf{q}_{2}, \nu_{2}\right)=\frac{3 N}{\left(2 \varepsilon_{\mathrm{F}}\right)^{2}} \gamma_{3}\left(q_{1}, q_{2},-t \mid \frac{\overline{\nu_{1}}}{q_{1}}, \frac{\overline{\nu_{2}}}{q_{2}}\right), \quad\left(\nu_{1}, \nu_{2}>0\right), \\
\Gamma_{3}\left(\mathbf{q}_{1}, \nu_{1} ;-\mathbf{q}_{2},-\nu_{2}\right)=\frac{3 N}{\left(2 \varepsilon_{\mathrm{F}}\right)^{2}} \gamma_{3}\left(q_{1},-q_{2}, t \mid \frac{\overline{\nu_{1}}}{\left.\overline{q_{1}}, \frac{\overline{\nu_{2}}}{q_{2}}\right),} \quad\left(\nu_{1}, \nu_{2}>0\right),\right. \\
\Gamma_{3}\left(\mathbf{q}_{1}, \nu_{1} ; \mathbf{q}_{2},-\nu_{2}\right)=\frac{3 N}{\left(2 \varepsilon_{\mathrm{F}}\right)^{2}} \gamma_{3}\left(q_{1},-q_{2},-t \mid \frac{\overline{\nu_{1}}}{q_{1}}, \overline{\overline{\nu_{2}}} \overline{q_{2}}\right), \quad\left(\nu_{1}, \nu_{2}>0\right) .
\end{aligned}
$$




\section{References}

1. Bohm D., Pines D., Phys. Rev., 1951, 82, No. 4, 625-634.

2. Bohm D., Pines D., Phys. Rev., 1952, 85, No. 2, 338-353.

3. Bohm D., Pines D., Phys. Rev., 1953, 92, No. 3, 609-625.

4. Pines D., Phys. Rev., 1953, 92, No. 3, 626-636.

5. Bohm D., Huang K., Pines D., Phys. Rev., 1957, 107, No. 1, 71-80.

6. Nozieres P., Pines D., Phys. Rev., 1958, 111, No. 2, 442-454.

7. Yukhnovskii I.R., Ukr. Fiz. Zhurn., 1964, 9, No. 7, 702-714 (in Russian).

8. Yukhnovskii I.R., Ukr. Fiz. Zhurn., 1964, 9, No. 8, 827-838 (in Russian).

9. Yukhnovskii I.R., Tsiganenko V.V., Vavrukh M.V, Ukr. Fiz. Zhurn., 1965, 10, No. 2, 135-146 (in Russian).

10. Yukhnovskii I.R., Petrashko R.N., Teor. Mat. Fiz., 1973, 17, No. 1, 118-130 (in Russian).

11. Vavrukh M.V., Slobodyan S.B., Conden. Matt. Phys., 2005, 8, No. 3, 453.

12. Vavrukh M.V., Visnik Lviv. Univer. seriya fizichna, 1968, No. 3, 26-33 (in Ukrainian).

13. Vavrukh M.V., Slobodyan S.B., Journ. Phys. Stud., 2003, 7, No. 3, 275-282 (in Ukrainian).

14. Vavrukh M., Tyshko N., Koval' S., Kushtay Ya., Journ. Phys. Stud., 2003, 7, No. 4, 375-387 (in Ukrainian).

15. Vavrukh M., Krokhmalskii T., Phys. Stat. Sol. (b), 1991, 168, 519-532.

16. Hwa R.C., Teplitz V.L. Homology and Feynman integrals. W.A. Benjamin, NewYork/Amsterdam, 1966.

\section{Тричастинкова кореляційна функція в електрон-плазмонній моделі}

\section{М.В.Ваврух, С.Б.Слободян, Н.Л.Тишко}

Львівський національний університет імені Івана Франка, кафедра астрофізики, 79005 Львів, вул. Кирила і Мефодія, 8

Отримано 3 серпня 2005 р.

Наведено спосіб розрахунку $\boldsymbol{n}$-частинкових електронних кореляційних функцій електрон-плазмонної моделі, яка була запропонована авторами раніше для опису сильно неідеальної електронної рідини. Розраховано і представлено в елементарних функціях тричастинкову динамічну кореляційну функцію. Досліджено її відмінності від аналогічної кореляційної функції звичайного базисного підходу у теорії електронної рідини.

Ключові слова: електронна рідина, плазмові коливання, $n$-частинкові динамічні кореляційні функції, оператор переходу, колективні змінні

PACS: 05.30.FK 